\title{
STABILITY AND TRANSVERSALITY
}

\author{
BY ROBERT D. MAY ${ }^{1}$
}

Communicated by Shlomo Sternberg, June 27, 1973

1. Introduction. Let $N$ and $P$ be $C^{\infty}$ manifolds of dimensions $n$ and $p$ and let $C^{\infty}(N, P)$ denote the space of all $C^{\infty}$ mappings $f: N \rightarrow P$ with the fine $C^{\infty}$ topology [2, II, p. 259]. A mapping $f \in C^{\infty}(N, P)$ may be stable in either the $C^{\infty}[2, \mathrm{II}]$ or topological [3] sense. In this paper we state certain results connecting these two concepts of stability. In a related development we also outline a procedure for showing that topologically stable mappings satisfy certain transversality conditions. All of the results given here are based on our thesis [4] to which we refer for proofs and further details.

2. A conjecture. It is clear that any $C^{\infty}$ stable mapping is also topologically stable, but the converse is false in general. In fact for $N$ compact Mather has shown that the topologically stable mappings are always dense in $C^{\infty}(N, P)$ [3], while the $C^{\infty}$ stable mappings are dense if and only if $n, p$ lie in a certain "nice" range [2, VI]. However, one may still conjecture the following:

(2.1) If $N$ is compact and $n$, p lie in the "nice" range, then any topologically stable mapping

is also $C^{\infty}$ stable.

$$
f: N \rightarrow P
$$

In [4] we verify the above conjecture for the comparatively simple cases $p>2 n$ ("Whitney embedding" range) and $p=1$ ("functions"). We obtain related results for a more substantial range of dimensions by introducing a "uniform stability" condition.

Definition. $f \in C^{\infty}(N, P)$ is uniformly stable provided that for any family

$$
F:\left(\boldsymbol{R}^{K}, 0\right) \rightarrow\left(C^{\infty}(N, P), f\right)
$$

of maps (parameterized by $\boldsymbol{R}^{K}$, any $K>0$ ) for which the associated map

$$
\tilde{F}: N \times R^{K} \rightarrow P \times R^{K}
$$

AMS (MOS) subject classifications (1970). Primary 58C25.

${ }^{1}$ This research was partially supported by NSF grant GP-31359-X-1. 
is $C^{\infty}$, there exists a neighborhood $U$ of $0 \in \boldsymbol{R}^{K}$ and homeomorphisms $h_{N}, h_{P}$ for which the following diagram commutes:

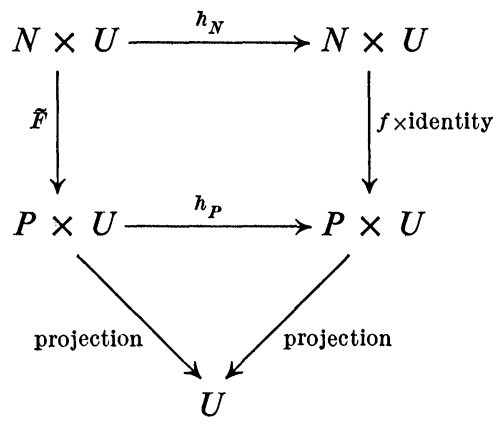

A major result of $[4]$ can now be stated as follows:

THeOREM 2.2. Let $N$ be compact and assume $n>p, p<7, n<2(n-p+2)$. Let $f \in C^{\infty}(N, P)$ be

(a) topologically stable, and

(b) in the interior of the set of uniformly stable maps.

Then fis $C^{\infty}$ stable.

3. A more general problem. In view of Mather's characterization $[2, \mathrm{~V}]$ of $C^{\infty}$ stable mappings, the major task in proving Theorem 2.2 is to show that $f$ is transverse to each orbit in $J^{p+1}(N, P)$ of the group $\mathscr{K}^{p+1}$ of $[2, \mathrm{IV}]$. We are then led to a more general question treated in [4]. Let $J^{k}(n, p)$ be the space of $k$-jets at 0 of $C^{\infty}$ mappings $f:\left(\boldsymbol{R}^{n}, 0\right) \rightarrow$ $\left(\boldsymbol{R}^{p}, 0\right)$, and let $\Sigma$ be a submanifold of $J^{k}(n, p)$ which is invariant under the group $\mathscr{K}^{k}$. Then for any $N, P$ we have an associated subbundle $\Sigma(N, P) \subseteq J^{k}(N, P)$ with fiber $\Sigma$. We would like to know whether for any compact $N$ and topologically stable $f: N \rightarrow P$ we have $j^{k} f$ transverse to $\Sigma(N, P)$. In the next sections we outline the procedure, of [4] for attacking this problem.

4. Topological transversality. We first replace transversality to $\Sigma(N, P)$ by a more "topological" concept. Given $\Sigma \subseteq J^{k}(N, P)$ and $f \in C^{\infty}(N, P)$, define $\Sigma(f) \subseteq N$ by $\Sigma(f)=\left(j^{k} f\right)^{-1}[\Sigma]$. Also for any submanifold $\Sigma \subseteq J^{k}(N, P)$ define

$$
\operatorname{cod} \Sigma=\text { dimension } J^{k}(N, P)-\text { dimension } \Sigma .
$$

Definition. Let $\Sigma$ be a submanifold of $J^{k}(N, P)$ and let $f \in C^{\infty}(N, P)$. Then $f$ is topologically transverse to $\Sigma$ at $x \in N$ if either $x \notin \Sigma(f)$, or

Case A. $n>\operatorname{cod} \Sigma$ and there exist neighborhoods $U$ of $x, W$ of $f$, 
such that $\Sigma(g) \cap U$ is a topological manifold of dimension $n-\operatorname{cod}(\Sigma)$ for all $g \in W$, or

Case B. $n=\operatorname{cod}(\Sigma)$ and there exist neighborhoods $U$ of $x, W$ of $f$, such that $\Sigma(g) \cap U$ is a single point for all $g \in W$.

It follows from familiar properties of transversal maps that transversality $\Rightarrow$ topological transversality for any $f$ and $\Sigma$. The converse is unclear, but we have proved the following [4]:

Proposition 4.1. Let $\Sigma$ be a $\mathscr{K}^{k}$-invariant submanifold of $\mathrm{J}^{k}(n, p)$ which is contained in a Boardman singularity [1] of the form

$$
\Sigma^{i_{1}, i_{2}, \cdots, i_{k}}, \quad i_{k}=0 .
$$

Then a map $f: N \rightarrow P$ is transverse to $\Sigma(N, P)$ if and only if $f$ is topologically transverse to $\Sigma(N, P)$.

Proposition 4.2. Let $\Sigma^{i} \subseteq J^{1}(n, p)$ be a first order Boardman singularity [1]. Then a map $f: N \rightarrow P$ is transverse to $\Sigma^{i}(N, P)$ if and only iff is topologically transverse to $\Sigma^{i}(N, P)$.

5. Germ classes. Let $C^{\infty}(n, p)$ denote the set of germs $[f]$ at 0 of $C^{\infty}$ mappings $f:\left(\boldsymbol{R}^{n}, 0\right) \rightarrow\left(\boldsymbol{R}^{p}, 0\right)$. [f], $[g] \in C^{\infty}(n, p)$ are $C^{\infty}$ (respectively, topologically) equivalent if there exist diffeomorphisms (respectively, homeomorphisms) $h_{n}:\left(\boldsymbol{R}^{n}, 0\right) \rightarrow\left(\boldsymbol{R}^{n}, 0\right), h_{p}:\left(\boldsymbol{R}^{p}, 0\right) \rightarrow\left(\boldsymbol{R}^{p}, 0\right)$ such that $[f]=\left[h_{p}^{-1}<g \circ h_{n}\right]$. A subset $\Sigma \subseteq C^{\infty}(n, p)$ is a $C^{\infty}$ (respectively, topological) germ class if $[f] \in \Sigma \Rightarrow[g] \in \Sigma$ for any $[g]$ which is $C^{\infty}$ (respectively, topologically) equivalent to [f]. If $\Sigma$ is a $C^{\infty}$ (or topological) germ class and $f \in C^{\infty}(N, P)$, we can define $\Sigma(f) \subseteq N$ by

$$
x \in \Sigma(f) \Leftrightarrow\left[\varphi \circ f \circ \psi^{-1}\right] \in \Sigma
$$

where $\psi:(U, x) \rightarrow\left(\boldsymbol{R}^{n}, 0\right)$ and $\varphi:(V, f(x)) \rightarrow\left(\boldsymbol{R}^{p}, 0\right)$ are local coordinates on $N, P$.

We call a $C^{\infty}$ germ class $\Lambda \subseteq C^{\infty}(n, p)$ generic if $\left\{f \in C^{\infty}(N, P) \mid \Lambda(f)=N\right\}$ is. dense in $C^{\infty}(N, P)$ for any $N, P$. For example the set of germs of all maps satisfying a countable number of transversality conditions will be generic by the Thom transversality theorem $[2, \mathrm{~V}]$.

In [4] we prove the following basic results relating topological transversality to the existence of appropriate topological germ classes. For these results we assume the source manifold $N$ is compact.

Proposition 5.1. Let $\Sigma$ be a $\mathscr{K}^{k}$-invariant submanifold of $J^{k}(n, p)$ and $\Sigma_{\text {Top }}$ a topological germ class in $C^{\infty}(n, p)$ such that $\Sigma(f)=\Sigma_{\text {Top }}(f)$ for any topologically stable mapping $f \in C^{\infty}(N, P)$. Then any topologically stable 
map $f \in C^{\infty}(N, P)$ is topologically transverse to $\Sigma(N, P)$ at every point $x \in N$.

Proposition 5.2. Let $\Sigma \subseteq C^{\infty}(n, p)$ be a closed $C^{\infty}$ germ class and $\Sigma_{\mathrm{Top}}$ a topological germ class. Assume there exists a generic class $\Lambda \subseteq$ $C^{\infty}(n, p)$ such that

(i) $\Lambda \cap \Sigma_{\text {Top }}=\Lambda \cap \Sigma$;

(ii) $\Lambda \cap \Sigma$ is dense in $\Sigma$;

(iii) for any $[g] \in \Lambda$ and any open $U$ containing $0 \in \boldsymbol{R}^{n}$, there exists an open $U^{\prime} \subseteq U, 0 \in U^{\prime}$, such that $U^{\prime} \cap \Sigma(g)$ is connected.

Then for any $N, P$ and topologically stable $f \in C^{\infty}(N, P)$, we have $\Sigma(f)=\Sigma_{\text {Top }}(f)$.

6. Summary. Our procedure for proving transversality properties of topologically stable mappings is then as follows: Given a $\mathscr{K}^{k}$-invariant submanifold $\Sigma \subseteq J^{k}(n, p)$, we write $\Sigma=\Sigma_{1}-\Sigma_{2}$, where $\Sigma_{1}, \Sigma_{2}$ denote closed, $\mathscr{K}^{k}$-invariant subsets of $J^{k}(n, p)$ and also the $C^{\infty}$ germ classes in $C^{\infty}(n, p)$ corresponding to $\Sigma_{1}, \Sigma_{2}$. We next find topological germ classes $\Sigma_{1, \text { Top }}, \Sigma_{2, \text { Top }}$ such that the hypotheses of Proposition 5.2 are satisfied by $\Sigma_{1}, \Sigma_{1, \text { Top }}$ and $\Sigma_{2}, \Sigma_{2, \text { Top }}$. It follows that

$$
\Sigma(f)=\Sigma_{1}(f)-\Sigma_{2}(f)=\left(\Sigma_{1, \mathrm{Top}}-\Sigma_{2, \mathrm{Top}}\right)(f)
$$

for any topologically stable $f$. But then by Proposition 5.1 any topologically stable $f$ is topologically transverse to $\Sigma$. Finally, if $\Sigma$ satisfies the conditions of Proposition 4.1 or 4.2 we have $f$ transverse to $\Sigma$ for any topologically stable $f$.

In [4] the above program is carried out for various $\Sigma$. For example we show

Proposition 6.1. Let $\Sigma^{i} \subseteq J^{1}(n, p)$ be a first order Boardman singularity with $n \geqq \operatorname{cod} \Sigma^{i}$. Then for any $N, P, N$ compact, and any topologically stable $f \in C^{\infty}(N, P)$, we have $f$ transverse to $\Sigma^{i}(N, P)$.

Also, for the range of dimensions considered in Theorem 2.2 we use the above technique to show that any topologically stable $f \in C^{\infty}(N, P)$ is transverse to $\Sigma(N, P)$ for any $\mathscr{K}^{p+1}$-orbit $\Sigma \subseteq J^{p+1}(n, p)$, provided $N$ is compact and $n \geqq \operatorname{cod} \Sigma$. (The uniform stability condition (b) of Theorem 2.2 is then used only to show that $j^{p+1} f \cap \Sigma=\varnothing$ for those $\Sigma$ with $n<\operatorname{cod} \Sigma$.)

REMARK. When $N$ is not compact, a simple counterexample given in [4] shows that Theorem 2.2 (and Proposition 6.1) fail to hold even for proper mappings. However, analogous results are obtained in [4] for the noncompact case by replacing the condition of topological stability by that of $\varepsilon$-stability. 


\section{BIBLIOGRAPHY}

1. J. M. Boardman, Singularities of differentiable mappings, Inst. Hautes Études Sci. Publ. Math. 33 (1967), 21-57. MR 37 \#6945.

2. J. N. Mather, Stability of $C^{\infty}$ mappings. II, IV, V, VI, Ann. of Math. (2) 89 (1969), 254-291; Inst. Hautes Études Sci. Publ. Math. No. 37 (1969), 223-248; Advances in Math. 4 (1970), 301-336; Proceedings of the Liverpool Singularities-Symposium, 1 (1969/70), Lecture Notes in Math., vol. 192, Springer, Berlin, 1971, pp. 207-253. MR 41 \#4582; 43 \#1215b, c; 45 \#2747.

3. - Stratifications and mappings, Proceedings of Dynamical Systems, Salvadore, Brazil, July 1971 (to appear).

4. R. D. May, Transversality properties of topologically stable mappings, Ph.D. thesis, Harvard University, Cambridge, Mass., 1973.

Department of Mathematics, Harvard University, Cambridge, Massachusetts 02138

Current address: Department of Mathematics, University of Utah, Salt Lake City, Utah 84112 